\title{
TITLE:
}

\section{Cold-neutron interferometer of the Jamin type}

AUTHOR(S):

Kitaguchi, M; Funahashi, H; Nakura, T; Hino, M; Shimizu, HM

\section{CITATION:}

Kitaguchi, M ...[et al]. Cold-neutron interferometer of the Jamin type. PHYSICAL REVIEW A 2003, 67(3): 033609.

ISSUE DATE:

2003-03

URL:

http://hdl.handle.net/2433/39824

RIGHT:

Copyright 2003 American Physical Society 
PHYSICAL REVIEW A 67, 033609 (2003)

\title{
Cold-neutron interferometer of the Jamin type
}

\author{
M. Kitaguchi,* H. Funahashi, and T. Nakura \\ Department of Physics, Kyoto University, Kyoto 606-8502, Japan \\ M. Hino \\ Research Reactor Institute, Kyoto University, Kumatori, Osaka 590-0494, Japan
}

H. M. Shimizu

RIKEN, 2-1 Hirosawa, Wako, Saitama 351-0198, Japan

(Received 30 August 2002; published 26 March 2003)

\begin{abstract}
A cold-neutron interferometer using four independent multilayer mirrors is demonstrated. We have observed interference fringes with a contrast of $60 \%$. The interferometer is based on a pair of devices using etalons with an air gap of $9.75 \mu \mathrm{m}$ in spacing. The optical system is equivalent to the Jamin-type interferometer, which allows much larger spatial separation between the two coherent beams than the previous type of multilayer interferometer to broaden the applicability of neutron interferometry.
\end{abstract}

DOI: 10.1103/PhysRevA.67.033609

PACS number(s): 03.75.Dg, 03.65.Ta

Neutron interferometry is a powerful technique for studying fundamental physics. Numerous interesting experiments [1] have been performed since the first successful test of a single-crystal neutron interferometer [2]. However, the single-crystal interferometer is inherently unable to deal with a neutron that has a wavelength longer than twice its lattice constant. In order to investigate problems of fundamental physics, including tests of quantum measurement theories and searches for non-Newtonian effects of gravitation, the interferometry of cold neutrons is extremely important, since the sensitivity of the interferometer for a small interaction increases with neutron wavelength. Several attempts have been made to develop neutron interferometers for cold neutrons and very cold neutrons [3]. Interference fringes are given as a function of the relative phase. When there is an interaction energy difference $\Delta E$ between the two paths, the relative phase is written as

$$
\Delta \phi=2 \pi \frac{m \lambda L}{h^{2}} \Delta E,
$$

where $m$ is the neutron mass, $\lambda$ is the neutron wavelength, and $L$ is the interaction path length. A large interferometer scale also has the advantage of increasing the sensitivity to small interactions.

Using multilayer mirrors, a large-dimensional coldneutron interferometer was demonstrated [4]. The multilayer mirror is one of the most useful devices in cold-neutron optics. A multilayer of two materials with different potentials is understood as a one-dimensional crystal that is suitable for Bragg reflection of long-wavelength neutrons. A magnetic mirror, in which one of the two materials is ferromagnetic, works as a reflective mirror or as a transparent mirror depending on the polarity of the neutrons. Neutron spin interferometer [5] using magnetic mirrors, which is one of polar-

\footnotetext{
*Also at RIKEN, 2-1 Hirosawa, Wako, Saitama 351-0198, Japan. Electronic address: kitaguch@nh.scphys.kyoto-u.ac.jp
}

ization interferometer, enables us to study the spin-dependent interaction and to carry out more precision experiments, due to its higher contrast, than the original type of multilayer interferometer. Some remarkable experiments have been carried out with multilayer interferometers using magnetic mirrors, for example, double Stern-Gerlach experiments [6] and delayed choice experiments [7]. The range of application was, however, limited due to the small spatial separation between the two coherent beams. A cold-neutron interferometer with large spatial separation enables us to carry out much higher precision measurements and new types of experiment. With the large separation, we can insert some devices into the gap between the paths of the interferometer, for example, to investigate the topological nature of the Aharonov-Casher (AC) effect [8]. We can also measure more precisely the interaction that provides the relative phase $\Delta \phi$ depending on the area enclosed by the two beam paths [9]. The aim of our development is to increase the spatial beam separation of a multilayer interferometer in order to broaden the applicability of neutron interferometry.

The cold-neutron interferometer, using multilayer mirrors [4] and the neutron spin interferometer [5], employs a pair of "pairmirrors" or "spinsplitters". Each of them consists of a pair of multilayer mirrors and an intermediate monolayer. A pairmirror or a spinsplitter shifts the two coherent beams; however, the beams overlap each other (Fig. 1). Because the pairmirrors and spinsplitters are fabricated by the vacuum
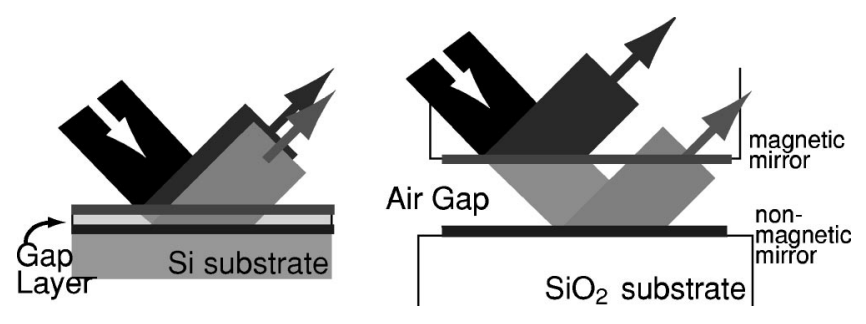

FIG. 1. Conventional spinsplitter (left) and beam splitting etalon (right). The present etalon can make larger spatial separations between two waves than a conventional spinsplitter. 

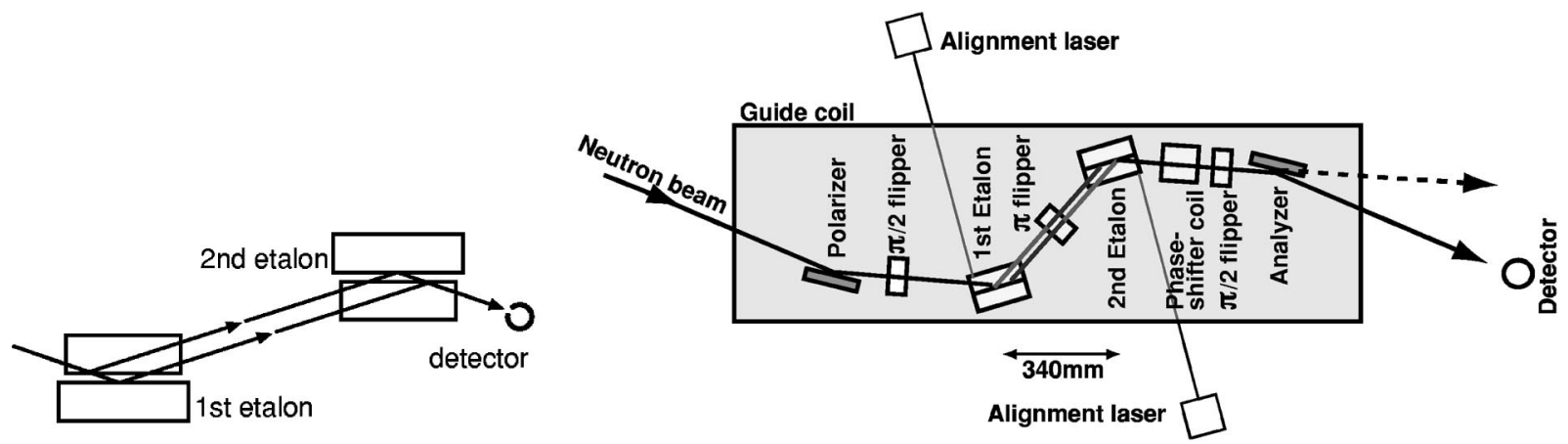

FIG. 2. Setup of the Jamin-type interferometer using etalon plates. The first beam splitting etalon (BSE) spatially separates up- and down-spin components into two parallel waves, and the two waves are spatially superposed on each other on the second BSE.

evaporation method, the gap between the two mirrors is of the order of $1 \mu \mathrm{m}$. It is not thick enough to separate the two beams spatially. We have used etalons in order to enlarge the spatial separation between the two coherent waves of the interferometer (Fig. 1). An etalon consists of two parallel planes finished with high precision. We can purchase special etalons with planes smooth enough to be used as substrates of neutron multilayer mirrors. By depositing multilayer mirrors on the parallel planes, such an etalon splits a neutron beam spatially.

Our experimental setup is shown in Fig. 2. It contains a pair of beam splitting etalons (BSEs). In the viewpoint of geometrical optics, the pair of BSEs is equivalent to a Jamin interferometer, which is the oldest type of interferometer for practical use in the history of optics. The polarizer and $\pi / 2$ flipper both provide the superposition of the spin-up and spin-down components. A magnetic neutron mirror deposited on one of the BSE planes reflects only the spin-up component and a nonmagnetic neutron mirror on the other plane reflects the spin-down component that is transmitted through the magnetic mirror. The BSE separates up- and down-spin components spatially into two parallel paths. The waves are spatially superposed on each other on the second BSE. The magnetic field provided by the phase-shifter coil gives the relative phase between the two components. By scanning the phase-shifter-coil current, interference fringes are observed through the $\pi / 2$ flipper and the analyzer mirror.

The present etalon made of fused silica has a spacing of $9.75 \mu \mathrm{m}$ and a diameter of $42 \mathrm{~mm}$. The mirrors were deposited in a diameter of $20 \mathrm{~mm}$ on a clear aperture of $30 \mathrm{~mm}$ diameter. The rms roughness of the present etalon plate is less than $3 \AA$ (SLS Optics). The etalon has matched front surfaces of $\lambda_{\mathrm{He}-\mathrm{Ne}} / 150$ in parallel. The mirrors were fabricated by the vacuum evaporation method at KURRI. The magnetic mirror has eight bilayers made of permalloy 45 $\left(\mathrm{Fe}_{55} \mathrm{Ni}_{45}\right)$ and germanium. The nonmagnetic mirror also has eight bilayers made of nickel and titanium. Both multilayers have an effective lattice constant of about $240 \AA$. The distance between the two BSEs was $340 \mathrm{~mm}$.

The experiment was performed using the cold-neutron beam line "MINE2" at the JRR-3M reactor in JAERI. The beam had a wavelength of $8.8 \AA$ and a bandwidth of $2.4 \%$ in full width at half maximum. We observed interference fringes with a contrast of $60 \%$ by scanning the phase-shifter- coil current (Fig. 3). Background counts were about 0.05 counts per second. In the present measurements, irregular drift of phase about $0.01 \mathrm{rad} / \mathrm{min}$ was found, which might be due to the rigidity of the present prototype. This drift is taken into account as a systematic error in the following discussion, but it does not affect our conclusion.

We measured the phase shift of the interferogram when the relative angle between the two BSEs changed (Fig. 4). The reflectivity of the mirrors on the etalon remains high in our narrow scanning range. The interferograms have maximum contrast point (echo point). The echo-point shift was also observed in the reverse direction of the phase shift of the interferogram (Fig. 5). As described below, we can understand that these two responses to the change of relative angle between the two BSEs result from the interference of two paths as shown in Fig. 2.

When we use a monochromatic beam with a wave number of $k_{0}$ and the standard deviation of Gaussian distribution $\sigma_{k}$, the relative phase between the two paths $\phi$ can be expanded as

$$
\begin{aligned}
\phi(k, \xi) & \simeq \phi\left(k_{0}, \xi\right)+\left.\frac{\partial \phi}{\partial k}\right|_{k=k_{0}}\left(k-k_{0}\right) \\
& \equiv \phi_{0}(\xi)+L_{0}(\xi)\left(k-k_{0}\right),
\end{aligned}
$$

where $\xi$ is a set of parameters of the phase shifters. Using Eq. (2), we can describe interferogram fringes as

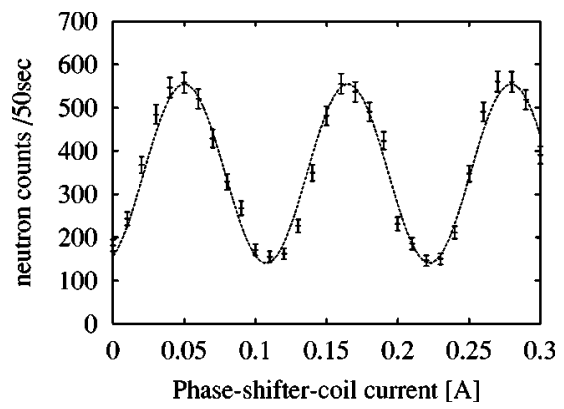

FIG. 3. Interference fringes resulting from scan of phase-shiftercoil current around the maximum contrast point. The contrast of fringes was $60 \%$. 


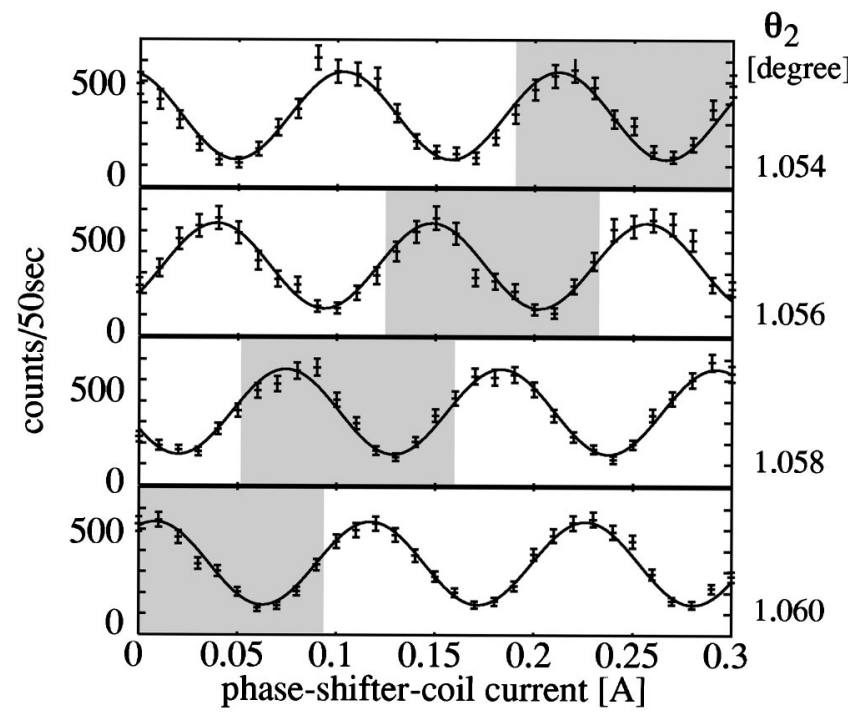

FIG. 4. Phase shift of the interferogram when the relative angle between two BSEs changes by $0.002^{\circ}$. The incident angle of the first BSE $\theta_{1}$ is fixed at $1.05^{\circ}$.

$$
I=\frac{1}{2}+\frac{a}{2} \exp \left\{-\frac{1}{2}\left(\sigma_{k} L_{0}\right)^{2}\right\} \cos \left(\phi_{0}\right),
$$

where $a$ is a factor of the contrast [10]. In an ideal case, $a$ $=1$, the contrast is maximized. Interference fringes oscillate with $\phi_{0} . L_{0}$ is the optical path difference between the two paths and $L_{0}$ of zero makes an "echo point." The relative phase provided by a pair of BSEs, $\phi_{\mathrm{E}}$, is expressed using the distance between the two mirrors $D$, the incident angle of the first BSE $\theta_{1}[\mathrm{rad}]$, and that of the second $\theta_{2}[\mathrm{rad}]$ as

$$
\phi_{\mathrm{E}}=2 \pi \frac{2 D\left(\sin \theta_{2}-\sin \theta_{1}\right)}{\lambda} \simeq 2 D \delta \theta \frac{2 \pi}{\lambda} \equiv A_{\mathrm{E}} \delta \theta k,
$$

where $\delta \theta$ is the relative angle between the two BSEs $\left(\theta_{2}\right.$ $\left.-\theta_{1}\right)$. The relative phase provided by phase-shifter coil $\phi_{\mathrm{C}}$ is

$$
\phi_{\mathrm{C}}=\gamma H t \equiv A_{\mathrm{C}} j \frac{1}{k}
$$

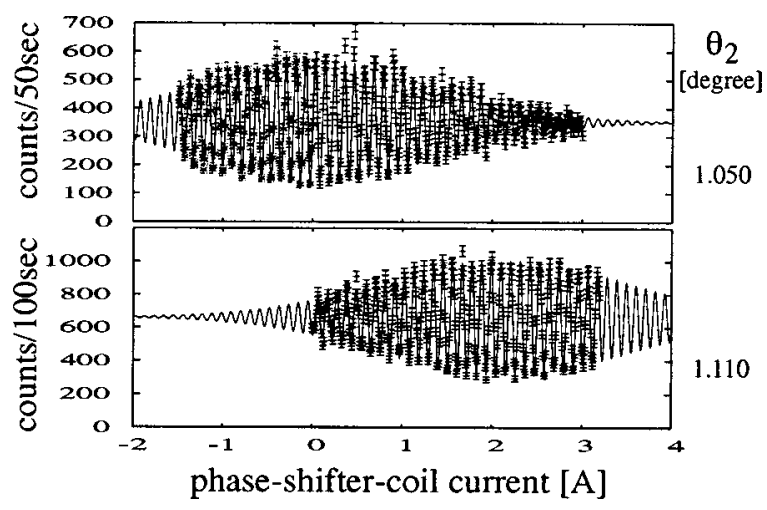

FIG. 5. Interference fringes resulting from wide range scanning of phase-shifter-coil current. The relative angle between the two BSEs was $0^{\circ}$ in the top panel and $0.06^{\circ}$ in the bottom panel. where $\gamma$ is the gyromagnetic ratio of neutron, $H$ is the magnetic field of the phase-shifter coil, $t$ is the time of flight through the coil (which is proportional to $k^{-1}$ ), $j$ is the current of the phase-shifter coil, and $A_{\mathrm{C}}$ is a conventional constant. $\phi_{\mathrm{E}}$ and $\phi_{\mathrm{C}}$ differ in $k$ dependence. The sum of the relative phase between the two paths is

$$
\begin{aligned}
\phi(k, \delta \theta, j)= & \phi_{\mathrm{E}}+\phi_{\mathrm{C}} \\
\simeq & \left\{A_{\mathrm{E}} \delta \theta k_{0}+A_{\mathrm{E}} \delta \theta\left(k-k_{0}\right)\right\} \\
& +\left\{\frac{A_{\mathrm{C}} j}{k_{0}}-\frac{A_{\mathrm{C}} j}{k_{0}^{2}}\left(k-k_{0}\right)\right\} \\
= & \left(A_{\mathrm{E}} \delta \theta k_{0}+\frac{A_{\mathrm{C}} j}{k_{0}}\right)+\left(A_{\mathrm{E}} \delta \theta-\frac{A_{\mathrm{C}} j}{k_{0}^{2}}\right)\left(k-k_{0}\right) .
\end{aligned}
$$

Using the expression of Eq. (2), we find

$$
\begin{gathered}
\phi_{0}=A_{\mathrm{E}} \delta \theta k_{0}+\frac{A_{\mathrm{C}}}{k_{0}} j, \\
L_{0}=A_{\mathrm{E}} \delta \theta-\frac{A_{\mathrm{C}}}{k_{0}^{2}} j .
\end{gathered}
$$

Equation (7) shows that a period of the interferogram is $\left(2 \pi k_{0} / A_{\mathrm{C}}\right)[\mathrm{A}]$ when $\delta \theta$ is fixed, and that a phase of the interferogram shifts by $-\left(A_{\mathrm{E}} k_{0} / 2 \pi\right) \Delta \theta$ cycles when changing $\delta \theta$ by $\Delta \theta$. On the other hand, Eq. (8) shows that an echo point shifts by $+\left(A_{\mathrm{E}} k_{0} / 2 \pi\right) \Delta \theta$ cycles, which is a quantity equal to the reverse direction of the phase shift of the interferogram. Through the changes of $\theta_{2}$ from $1.050^{\circ}$ to $1.060^{\circ}$, a phase of the interferogram was found to shift as

$$
-\frac{A_{\mathrm{E}} k_{0}}{2 \pi} \Delta \theta=-(1.94 \pm 0.04) \times 10^{4} \Delta \theta
$$

From Fig. 5 the echo-point shift was evaluated as

$$
+\frac{A_{\mathrm{E}} k_{0}}{2 \pi} \Delta \theta=+(1.93 \pm 0.04) \times 10^{4} \Delta \theta
$$

The expected value of $\left(A_{\mathrm{E}} k_{0} / 2 \pi\right)=\left(2 D / \lambda_{0}\right)$ is $2.17 \times 10^{4}$. This discrepancy is understood by the refraction of the etalon substrate of BSE through which the neutrons are transmitted. When we correct for the refraction by multiplying Eq. (4) by $\left[\sin \theta_{2} / \sqrt{\left(1-n^{2}\right)+\sin ^{2} \theta_{2}}\right]=0.89$, the correcting value of the coefficient $A_{\mathrm{E}} k_{0} / 2 \pi$ becomes $1.94 \times 10^{4}$, where $n$ is the refractive index of the etalon substrate.

In conclusion, we remark that the observed interferograms were consequent upon the two optical paths of the Jamintype interferometer using a pair of "beam splitting etalons," and that this represents a breakthrough in neutron interferometry by using four multilayer mirrors deposited on independent substrates. The four mirrors are aligned with high precision on the parallel planes of the etalons. The present results have demonstrated the feasibility of developing a large-dimensional interferometer with a large spatial path 
separation for long-wavelength neutrons. We are continuing the test experiments using new air-spaced etalons with a spacing of $200 \mu \mathrm{m}$ thickness. We plan to increase the etalon air gap spacing to $1 \mathrm{~mm}$. The results have qualified the flatness and roughness of the present etalon plates as substrates good enough to be used for neutron mirrors, which cause no serious distortion of wave front to compose an interferometer. By using such mirrors as mentioned above, we can now even arrange an optical system of Mach-Zehnder type.

The enlargement of path separation in the cold-neutron interferometer enables us to carry out high-precision measurements and different types of experiments, which have not, so far, been accomplished by other interferometers developed. We also plan some applications of the present type of interferometer. High-precision measurement of the topological Aharonov-Casher effect [8] and a gravitationally induced quantum interference [9] are interesting subjects to be carried out. In addition, a new type of quasielastic scattering spectrometer [11], which is equivalent to the neutron spinecho system (NSE), can be realized by using four BSEs arranged in $(++--)$ configuration. Such a spectrometer will be much smaller than conventional NSE. Using the BSEs with a gap of $200 \mu \mathrm{m}$ and neutrons of wavelength of $20 \AA$, the Fourier time of the spectrometer reaches about $300 \mathrm{~ns}$. The spectrometer has broad applicability for the study of slow dynamics including biophysics.

The authors gratefully acknowledge fruitful discussions with Professor T. Ebisawa, Professor S. Tasaki, and Professor Y. Otake. This work was supported by the interuniversity program for common use JAERI and KUR. This work was supported financially by a Grant-in-Aid for Scientific Research (Grant No. 12740149) of JSPS, the REIMEI Research Resources of JAERI, and Special Coordination Funds for Promoting Science and Technology of the Ministry of Education of Japanese Government. One of the authors (M.K.) was supported by the Junior Research Associates Program of RIKEN.
[1] H. Rauch and S. Werner, Neutron Interferometry (Oxford University Press, Oxford, 2000); J. Byrne, Neutron, Nuclei and Matter (Institute of Physics Publishing, London, 1994), Chap. 7; Mater Wave Interferometry, edited by G. Badurek, H. Rauch, and A. Zeilinger (North-Holland, Amsterdam, 1988).

[2] H. Rauch, W. Treimer, and U. Bonse, Phys. Lett. 47A, 369 (1974).

[3] A.I. Ioffe et al., Phys. Lett. 111, 373 (1985); M. Gruber et al., Phys. Lett. A 140, 363 (1989).

[4] H. Funahashi et al., Phys. Rev. A 54, 649 (1996).

[5] T. Ebisawa et al., Phys. Rev. A 57, 4720 (1998).

[6] Y. Otake et al., Proceedings of the International Symposium on Foundations of Quantum Mechanics, Tokyo, 1998, edited by Y.A. Ono and K. Fujikawa (Elsevier, 1999), p. 323.

[7] T. Kawai et al., Nucl. Instrum. Methods Phys. Res. A 410, 259 (1998).

[8] A. Cimmino et al., Nucl. Instrum. Methods Phys. Res. A 440, 579 (2000); A. Cimmino et al., Phys. Rev. Lett. 63, 380 (1989).

[9] S.A. Werner et al., Physica B \& C 151, 22 (1988); K.C. Littrell et al., Phys. Rev. A 56, 1767 (1997).

[10] Y. Morikawa and Y. Otake, Nuovo Cimento Soc. Ital. Fis., B 105B, 507 (1990).

[11] T. Ebisawa et al., Phys. Lett. A 259, 20 (1999). 